\title{
High-Efficiency Beam Extraction and Collimation Using Channeling in Very Short Bent Crystals
}

\author{
A. G. Afonin, ${ }^{1}$ V. T. Baranov, ${ }^{1}$ V. M. Biryukov, ${ }^{1}$ M. B. H. Breese, ${ }^{2}$ V. N. Chepegin,,${ }^{1}$ Yu. A. Chesnokov, ${ }^{1}$ V. Guidi ${ }^{3}$ \\ Yu. M. Ivanov, ${ }^{5}$ V. I. Kotov, ${ }^{1}$ G. Martinelli, ${ }^{4}$ W. Scandale, ${ }^{6}$ M. Stefancich, ${ }^{4}$ V. I. Terekhov, ${ }^{1}$ D. Trbojevic, ${ }^{7}$ \\ E. F. Troyanov, ${ }^{1}$ and D. Vincenzi ${ }^{4}$ \\ ${ }^{1}$ Institute for High Energy Physics, Protvino, 142281, Russia \\ ${ }^{2}$ Surrey University, Guildford, GU2 5XH, United Kingdom \\ ${ }^{3}$ Ferrara University, Department of Physics and INFN, I-44100, Italy \\ ${ }^{4}$ Ferrara University, Department of Physics and INFM, I-44100, Italy \\ ${ }^{5}$ Petersburg Nuclear Physics Institute, Gatchina, 188350, Russia \\ ${ }^{6}$ CERN, Geneva 23, CH-1211, Switzerland \\ ${ }^{7}$ Brookhaven National Laboratory, Upton, New York 11973
}

(Received 12 April 2001; published 14 August 2001)

\begin{abstract}
A silicon crystal was used to channel and extract $70 \mathrm{GeV}$ protons from the $\mathrm{U}-70$ accelerator with an efficiency of $85.3 \pm 2.8 \%$, as measured for a beam of $\sim 10^{12}$ protons directed towards crystals of $\sim 2 \mathrm{~mm}$ length in spills of $\sim 2 \mathrm{~s}$ duration. The experimental data follow very well the prediction of Monte Carlo simulations. This demonstration is important in devising a more efficient use of the U-70 accelerator in Protvino and provides crucial support for implementing crystal-assisted slow extraction and collimation in other machines, such as the Tevatron, RHIC, the AGS, the SNS, COSY, and the LHC.
\end{abstract}

DOI: 10.1103/PhysRevLett.87.094802

PACS numbers: $41.85 .-\mathrm{p}$

The technique of bent crystal channeling to steer particle beams, with applications in extraction from accelerator and in beam collimation, has progressed rapidly thanks to efforts at IHEP Protvino [1], CERN [2], and FNAL [3]. Beams of up to $10^{6}$ proton/s were extracted from CERN Super Proton Synchrotron and Tevatron by Si crystals of just $4 \mathrm{~cm}$ in length, with typical efficiencies on the order of $10 \%-20 \%$. It was predicted [4] that efficiency of crystal channeling extraction can be boosted to much higher values by multiple particle encounters with a shorter crystal installed in a circulating beam. There has also been experimental evidence that a multipass mechanism is responsible for efficient crystal extraction [5,6]. To clarify this mechanism a new experiment was started at IHEP at the end of 1997, with the intention of testing very short crystals and achieving very high efficiencies of extraction $[7,8]$.

The benefits of a crystal-assisted extraction are fourfold. In hadron colliders this mode of extraction can in general be made compatible with the colliding mode of operation. The time structure of the extracted beam is practically flat, since the extraction mechanism is resonance-free. The size of the extracted beam is smaller and more round than in a resonant extraction. Finally, polarized beams can be extracted without detrimental effects on the polarization.

The classic two-stage collimation system for loss localization in accelerators typically uses a small scattering target as a primary element and a bulk absorber as a secondary element [9]. The role of the primary element is to give a substantial angular kick to the incoming particles in order to increase the impact parameter on the secondary element, which is generally placed in the optimum position to intercept transverse or longitudinal beam halos. An amorphous primary target scatters the impinging particles in all possible directions. Ideally, one would prefer to use a "smart target" which kicks all particles in only one direction: for instance, only in the radial direction, only outward, and only into the preferred angular range corresponding to the center of the absorber (to exclude escapes). A bent crystal is the practical implementation for such a smart target: it traps particles and conveys them into the desired direction. Here, the random scattering process on single atoms of an amorphous target becomes the selective and coherent scattering on atomic planes of an aligned monocrystal.

These two possible applications of crystal channeling in modern hadron accelerators, extraction and halo collimation, can be exploited in a broad range of energies, from sub-GeV cases (i.e., for medical accelerators) to multi-TeV machines (for high-energy research). Indeed, several projects are in progress to investigate them. Crystal collimation is studied at RHIC (100-250 GeV) [10], considered for the Tevatron $(1000 \mathrm{GeV})$ [11] and the LHC [12] for the spallation neutron source (SNS) (1 GeV) [13], while crystal-assisted slow extraction is considered for Alternating Gradient Syncrotron (25 GeV) [14] and COSY $(1-2 \mathrm{GeV})$. In all cases, the critical issue is the channeling efficiency.

In the past two years, we demonstrated crystal channeling with $50 \%$ efficiency $[7,8]$. We also showed that, when properly aligned, these crystals could be efficiently used as primary collimators, thereby reducing by a factor of 2 the radiation level measured downstream of the collimation region of U-70. To continue our investigations, we installed and tested several new crystals in different straight sections of the U-70 ring. Three of them were produced by different manufacturers with a new shape. They were made with 
narrow strips of $\mathrm{Si}(111)$, about $40 \mathrm{~mm}$ long vertically and a fraction of a millimeter long in the radial direction. Their azimuthal length was only a few millimeters. They were bent by a metallic holder providing deflections of 0.8 to $1.5 \mathrm{mrad}$.

To make a crystal deflector this short (factor of $\sim 20$ shorter than the crystals used at CERN and FNAL), a new design was developed, (Fig. 1). The crystal strip has the shape of a saddle, being bent in both vertical and radial directions. This design was presented on page 85 in Ref. [1] and will be described in detail elsewhere [15].

Two crystals were assembled in Protvino: one $2 \mathrm{~mm}$ long was bent by $0.9 \mathrm{mrad}$, the other $4 \mathrm{~mm}$ long was bent by $1.5 \mathrm{mrad}$. The third crystal, $1.8 \mathrm{~mm}$ long, $0.8 \mathrm{mrad}$ bent, was prepared at the University of Ferrara and chemically polished at an optical level to remove the defects induced during diamond slicing. The two Russian crystals were used in extraction mode, while the Italian crystal was tested as a primary collimator. The three crystals were exposed to $70-\mathrm{GeV}$ proton beams and used to channel and extract halo particles.

Figure 2 illustrates the beneficial effect of crystals when used as primary collimators. It shows beam profiles in the radial direction downstream of the crystal as measured on the entry face of the collimator with a $15 \times 15$ channel secondary-emission profile meter [16]; the step size was $1.25 \mathrm{~mm}$, sensitivity $10^{8}$ proton/channel. Four cases are reported; in each case, the amount of incident beam was about the same. First, an amorphous collimator is used as primary target while the crystal is kept outside of the beam envelope. As expected, the beam profile

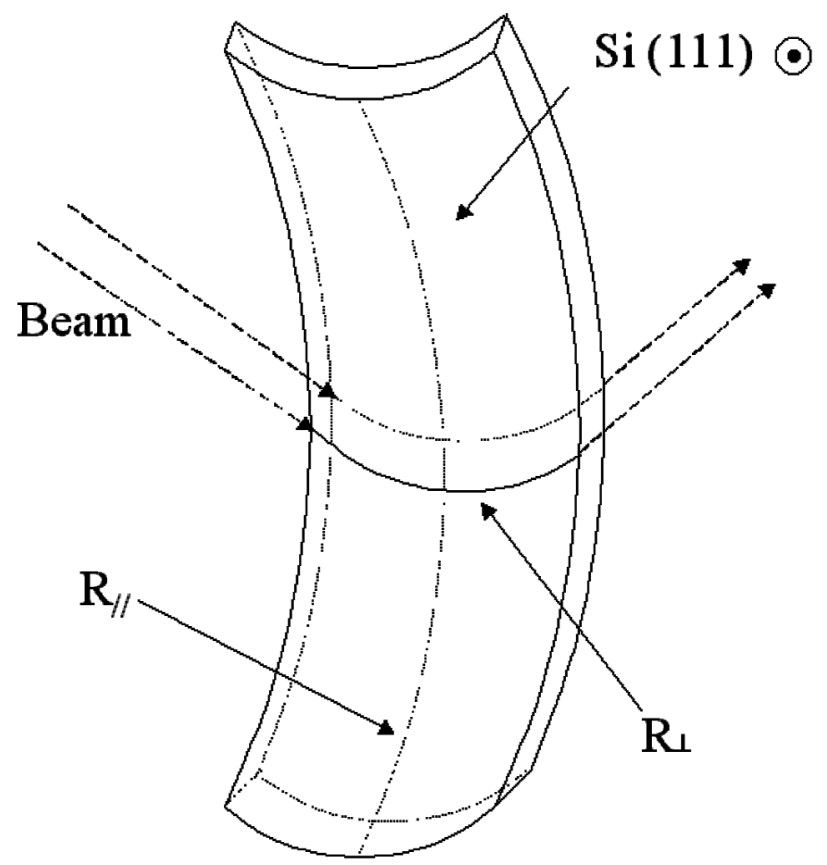

FIG. 1. Sketch of a crystal deflector shaped as a saddle due to curvature in both vertical and radial directions. This design was used to obtain $85 \%$ efficiency of extraction. is peaked at the collimator edge [Fig. 2(a)]. In the second case [Fig. 2(b)], the crystal is used as the primary scraper but is not aligned to the beam. Third, when properly aligned [Fig. 2(c)], the crystal channels most of the incoming beam into the depth of the collimator. In the fourth case, [Fig. 2(d)], the beam is simply kicked by a magnet towards the secondary collimator, while the crystal is retracted.

The extraction efficiency is given by the ratio of the extracted beam intensity as measured in the external beam
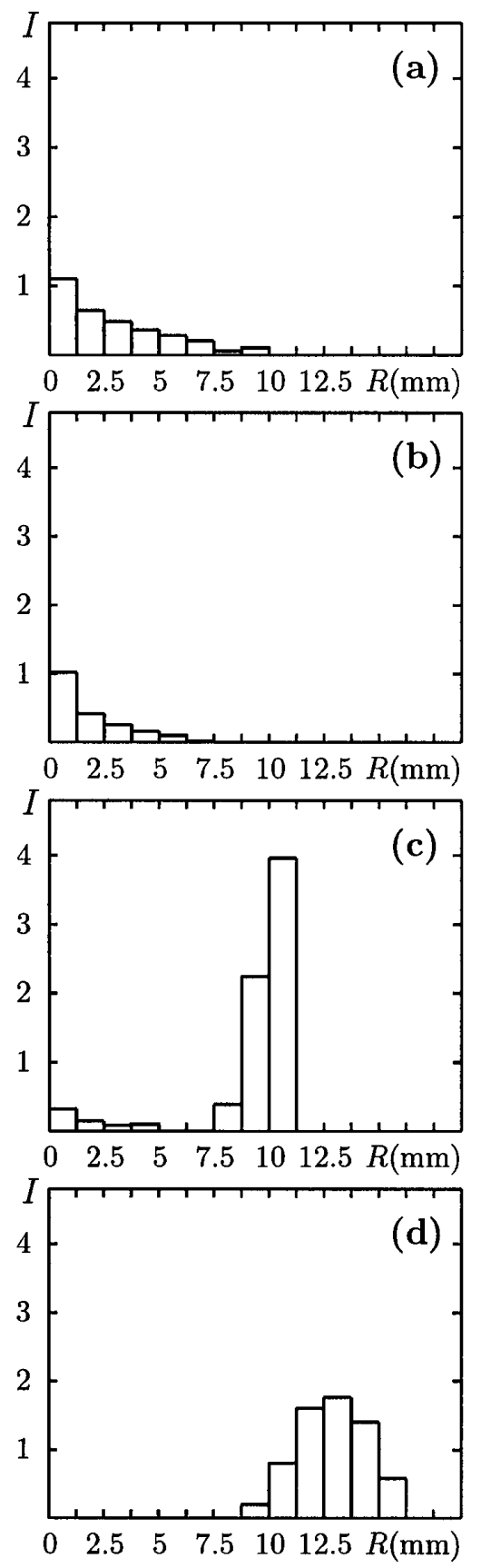

FIG. 2. Beam profiles measured on the collimator entry face: (a) crystal is out, beam scraped by collimator alone; (b) crystal is in the beam, but misaligned; (c) crystal is in the beam, aligned; (d) crystal is out, beam kicked by magnet. 


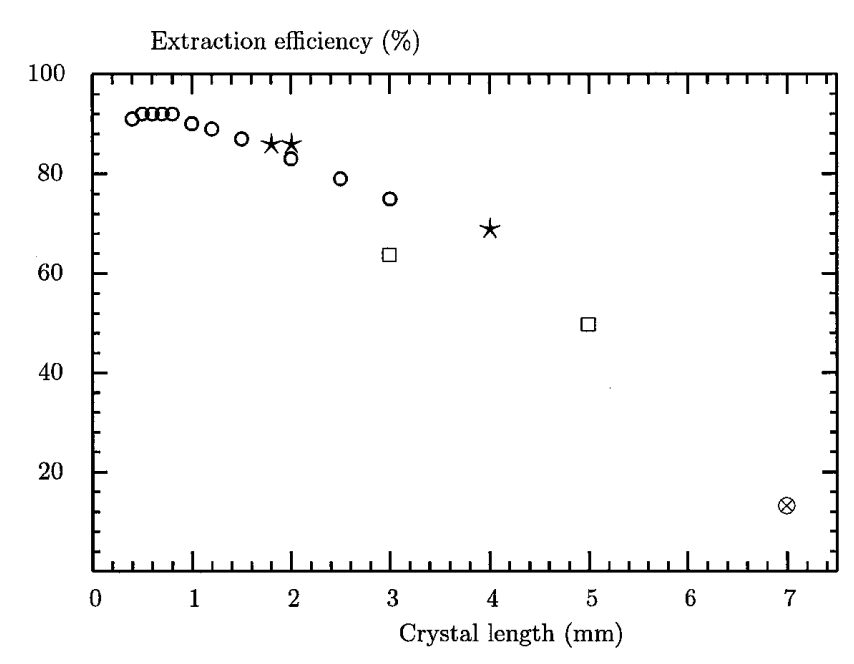

FIG. 3. Crystal extraction efficiency as measured for $70-\mathrm{GeV}$ protons. Recent results [( $)$, strips 1.8, 2.0, and $4 \mathrm{~mm}$, 1999-2000 [( $\square)$, O-shaped crystals 3 and $5 \mathrm{~mm}]$, and 1997 $[(\otimes)$, strip $7 \mathrm{~mm}]$. Also shown (०) is the Monte Carlo prediction for a perfect crystal with 0.9 mrad bending.

line to all the beam loss as measured in the entire ring. The diagnostic part of the experiment is described in some detail in $[7,8]$. The intensity of the extracted beam was measured with two independent monitors of secondary emission. The reduction in the circulating beam was measured with beam transformers. We obtained very high efficiencies in each of the three new crystals, namely, both the 1.8- and 2-mm-long crystals reached $85 \%$ efficiency, while the $4-\mathrm{mm}$-long crystal reached $68 \%$ efficiency. These striking results were obtained in a steady manner over many runs.

In Fig. 3 we plot the predicted [8] (by Monte Carlo code [17]) and the measured extraction efficiencies together with the data obtained earlier. In the measurements of 1997-2000 presented in Fig. 3 the bending angle of the crystals varied from 0.8 to $1.7 \mathrm{mrad}$ while the theoretical efficiency curve of Fig. 3 was calculated for 0.9 mrad. For $70-\mathrm{GeV}$ protons the crystal curvature in these cases is as yet far from the critical one, so we expect only a weak dependence of the efficiency on the bending angle. The agreement between measurements and simulations is excellent.

Besides the channeling efficiency, the ability to withstand a high beam intensity and the crystal lifetime are also important for a practical application. Crystals located in the region upstream of the U-70 cleaning area were irradiated with the entire circulating beam, spilled out in rather short time durations to simulate very dense halo collimation. We can measure precisely the beam intensity intentionally dumped into the crystal. However, we can only estimate with computer simulations the total amount of particle hits during a spill, since unchanneled protons are simply scattered and may continue to circulate in the ring, hitting the crystal many times. The number of hits per primary particle can vary from a few to $\sim 100$, depending on channeling efficiency, crystal length, and crystal alignment. We normally dumped $2 \times 10^{12}$ protons on a crystal per cycle. Computer analysis has shown that our crystals were irradiated up to $2 \times 10^{14}$ particles per spill of $\sim 1 \mathrm{~s}$ duration. When averaged over machine cycles, the irradiation rate was as high as $2 \times 10^{13}$ protons hits/s. Notice that this irradiation rate compares with the expected beam loss rate at the spallation neutron source. Indeed, the SNS accumulator ring should generate a $1-\mathrm{GeV}$ proton flux of $60 \times 2 \times 10^{14} \mathrm{~s}$. At the expected rate of beam loss of $0.1 \%-1 \%$ the halo flux will be $(1.2-12) \times 10^{13}$ proton $/ \mathrm{s}$. This seems to indicate that crystals may well tolerate high flux of protons as large as those expected to hit the SNS beam collimation system.

Another crystal, $5 \mathrm{~mm}$ long, was exposed for several minutes to even higher radiation flux. Beams of up to $10^{13}$ $70-\mathrm{GeV}$ protons (resulting in $\sim 10^{14}$ proton hits) were directed towards the crystal in spills of 50 ms every $9.6 \mathrm{~s}$. After this extreme exposure, the channeling properties of the crystal were tested in an external beam. The deflected beam observed with photoemulsion (Fig. 4) was perfectly normal, without breaks or significant tails eventually produced by dechanneled particles. Several crystals in use in U-70 have been exposed to high intensity beams for months, thereby accumulating very large integrated doses [8]. After the estimated irradiation of $\sim 10^{20} \mathrm{p} / \mathrm{cm}^{2}$ the initial channeling efficiency of about $43 \%$ was practically unaffected. This irradiation is still below the world's highest results [18,19]. The CERN experiment [19] showed that at $5 \times 10^{20}$ proton $/ \mathrm{cm}^{2}$ the crystal lost only $30 \%$ of its deflection efficiency, which means $\sim 100 \mathrm{yr}$ lifetime in the intense beam of NA48 experiment.

On the same location in the U-70 ring with the same $1.8 \mathrm{~mm}$ crystal of $\mathrm{Si}(111)$ positioned $\sim 20 \mathrm{~m}$ upstream of

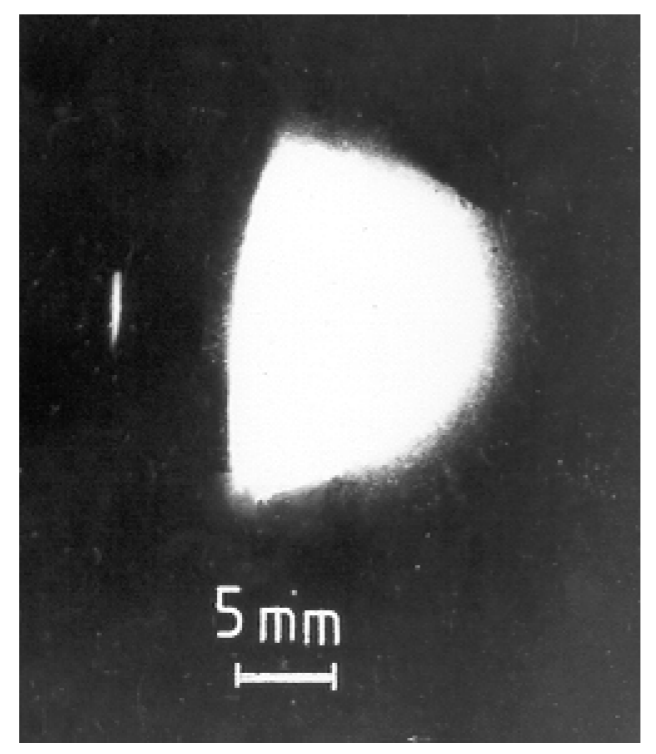

FIG. 4. Photograph of the deflected (left) and incident (right) beams as seen downstream of the crystal in an external beam test. Prior to the test, the crystal was exposed in the ring to $50 \mathrm{~ms}$ pulses of $10^{14}$ proton hits per pulse. No damage is seen. 


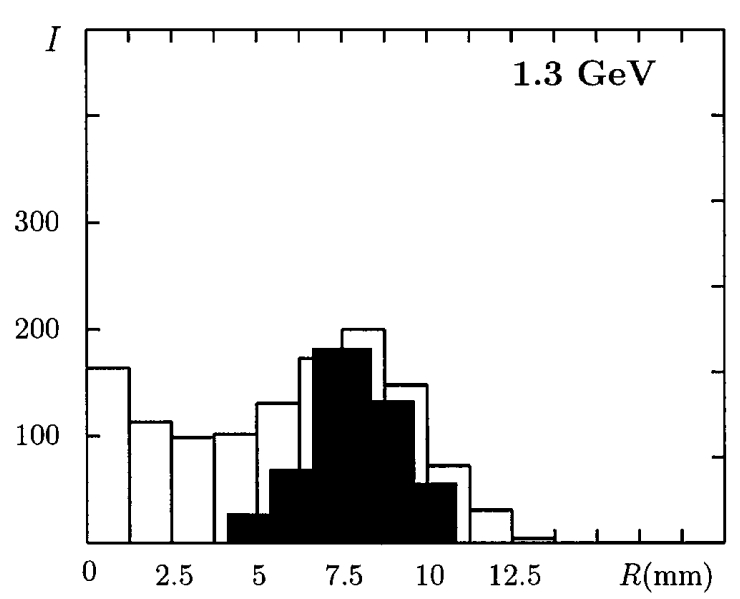

FIG. 5. Beam profile as measured on the collimator entry face with $1.3-\mathrm{GeV}$ protons. The black area shows the simulated profile of channeled protons.

the ring collimator, we repeated the crystal collimation experiment at the injection flattop of U-70, proton kinetic energy of $1.3 \mathrm{GeV}$. With the crystal aligned with the incoming halo particles, the radial beam profile at the collimator entry face showed a significant channeled peak far from the edge (Fig. 5). The expected width of the channeled peak is about 5 bins in the profile of Fig. 5, in agreement with observations. About half of the protons intercepted by the collimator jaw, were channeled there by a crystal; i.e., the crystal doubled the amount of particles intercepted by the jaw. As only part (about 34\%) of all particles scattered off the crystal reached the jaw, we estimate the crystal deflection efficiency as $15 \%-20 \%$. The observed figure of efficiency could be well reproduced in computer simulations. This figure is orders of magnitude higher than previous world data for low-GeV energy range. It is remarkable that the same crystal was efficiently channeling and really helping in cleaning halo particles both at $70 \mathrm{GeV}$ and at $1.3 \mathrm{GeV}$, thus demonstrating to be operational in a very wide energy range.

In summary, the crystal channeling efficiency has reached unprecedented high values both at top energy and at injection energy. The same 2-mm-long crystal was used to channel $70-\mathrm{GeV}$ protons with an efficiency of $85.3 \% \pm 2.8 \%$ during several weeks of operation and $1.32-\mathrm{GeV}$ protons with an efficiency of $15 \%-20 \%$ during some test runs. Crystals with a similar design were able to withstand radiation doses over $10^{20}$ proton $/ \mathrm{cm}^{2}$ and irradiation rates of $2 \times 10^{14}$ particles incident on crystal in spills of $\sim 2 \mathrm{~s}$ duration without deterioration of their performances.

The efficiency results well match the figures theoretically expected for ideal crystals. As simulations show, extraction and collimation with channeling efficiencies over $90 \%-95 \%$ is feasible. The obtained high figures provide crucial support for the ideas to apply this technique in beam cleaning systems, for instance, in RHIC and Tevatron. Earlier Tevatron scraping simulations [11] have shown that a crystal scraper can reduce accelerator-related backgrounds in $\mathrm{CDF}$ and D0 experiments by a factor of $\sim 10$. This year, the first experimental data is expected from RHIC where a crystal collimator [10] is installed. The technique presented here is potentially applicable also in LHC, for instance, to improve the efficiency of the LHC cleaning system by embedding bent crystals in the primary collimators [12].

This work was supported by INTAS-CERN Grant No. 132-2000, RFBR Grant No. 01-02-16229, and by the "Young researcher Project" of the University of Ferrara.

[1] V. M. Biryukov, Yu. A. Chesnokov, and V. I. Kotov, Crystal Channeling and its Application at High Energy Accelerators (Springer, Berlin, 1997).

[2] H. Akbari et al., Phys. Lett. B 313, 491 (1993).

[3] C. T. Murphy et al., Nucl. Instrum. Methods Phys. Res., Sect. B 119, 231 (1996).

[4] V. Biryukov, Nucl. Instrum. Methods Phys. Res., Sect. B 53, 202 (1991); A. Taratin et al., Nucl. Instrum. Methods Phys. Res., Sect. B 58, 103 (1991); V. Biryukov, Phys. Rev. E 52, 6818 (1995).

[5] X. Altuna et al., Phys. Lett. B 357, 671 (1995).

[6] R. A. Carrigan, Jr., Phys. Rev. ST Accel. Beams 1, 022801 (1998); R. A. Carrigan, Jr., et al., Report No. FERMILABPUB-99-186-E, 1999 (to be published).

[7] A. G. Afonin et al., Phys. Lett. B 435, 240 (1998); A. G. Afonin et al., in PAC'1999 Proceedings, New York (to be published); CERN Report No. LHC 99-2 (MMS) (to be published).

[8] V. I. Kotov et al., in EPAC'2000 Proceedings, Vienna (to be published).

[9] J. B. Jeanneret, Phys. Rev. ST Accel. Beams 1, 081001 (1998).

[10] D. Trbojevic et al., in EPAC Proceedings, Stockholm, 1988 (to be published).

[11] V. M. Biryukov, A. I. Drozhdin, and N. V. Mokhov, in PACS'99 Proceedings, New York (to be published); Fermilab Report No. Conf-99/072, 1999 (to be published).

[12] INTAS-CERN project "Crystal Technique for Halo Cleaning in the LHC" (approved 2001).

[13] N. Catalan-Lasheras et al., in EPAC'2000 Proceedings, Vienna (to be published).

[14] J. W. Glenn, K. A. Brown, and V. M. Biryukov, in PAC'01 Proceedings, Chicago (to be published).

[15] Yu. Chesnokov et al. (to be published).

[16] V. T. Baranov et al., in Proceedings of the XXVIIth Russian National Conference on Accelerators, Protvino, 2000 (to be published).

[17] V. Biryukov, Phys. Rev. E 51, 3522 (1995).

[18] S. I. Baker et al., Nucl. Instrum. Methods Phys. Res., Sect. B 90,119 (1994).

[19] A. Baurichter et al., Nucl. Instrum. Methods Phys. Res., Sect. B 164-165, 27 (2000). 